\title{
The importance of group B streptococci as human pathogens in the British Isles
}

\author{
I. A. HARPER ${ }^{1}$
}

From the Public Health Laboratory, Coventry and Warwickshire Hospital, Coventry

SYNOPSIS A case of meningitis due to a group B streptococcus in a newborn baby is described. Treatment was successful; it depended upon the bacteriological diagnosis and sensitivity testing. Only a few such cases are reported in the British literature, but this type of infection may be more common than these reports would suggest.

\section{History}

The baby, a male, was admitted to the Special Care Baby Unit, Coventry Maternity Hospital, 15 hours after delivery, because of twitching of the right eyelid and right arm, lasting five minutes; at this time his general condition was good. Two hours later there were convulsive movements of the right arm and face. Phenobarbitone, $15 \mathrm{mg}$, was given intramuscularly and this was repeated eight hours later. After a further 16 hours, four hours, and three hours similar convulsions followed, lasting for eight minutes and tending to become generalized; phenobarbitone dosage was increased to $15 \mathrm{mg}$ four hourly. At this time pyrexia of $39^{\circ} \mathrm{C}$ had developed and the baby appeared ill and jaundiced. Lumbar puncture was performed.

The mother, a primipara, had an uneventful pregnancy and labour lasted seven hours; vaginal examination was carried out when she was admitted to exclude prolapse of the cord. There was no premature rupture of the membranes; an episiotomy was carried out. Birth was four days overdue; the placenta was complete but gritty and infarcted although apparently normal otherwise and the lochia were normal. During her pregnancy, labour, and puerperium she was not pyrexial and there was no suggestion of genital tract or any other infection.

\section{INVESTIGATIONS}

The baby's cerebrospinal fluid was bloodstained and xanthrochromic and there was an excess of polymorphonuclear leucocytes (red blood cells/white blood cells $=30,000 / 560$ per cubic $\mathrm{ml}$ ); large 'Present address: Public Health Laboratory, New Cross Hospital, Wolverhampton

Received for publication 30 September 1970. numbers of lanceolate Gram-positive cocci were seen.

Streptococci were isolated from the cerebrospinal fluid, blood, and nasal secretions of the baby; similar streptococci were isolated also from the vagina of the mother two days after delivery.

Surface colonies were $0.5-1 \mathrm{~mm}$ in diameter, bluish-grey and glossy, after 24 hours' incubation aerobically at $37^{\circ} \mathrm{C}$ on horse blood agar; there was a narrow zone of incomplete haemolysis around the colonies. Stab inoculation into the medium resulted in colonies surrounded by a double zone of haemolysis, the inner zone being complete.

The organism grew on MacConkey's medium but not on $10 \%$ or $40 \%$ bile agar.

Sucrose, salicin, and trehalose were fermented; lactose, mannitol, sorbitol, glycerol, and inulin were not fermented. Arginine and hippurate were hydrolysed; aesculin was not hydrolysed.

In this laboratory the streptococci were extracted by Maxted's method (Maxted, 1948) and typed by precipitation methods (Lancefield, 1933); they belonged to group B. Dr M. T. Parker of the Cross Infection Reference Laboratory, Colindale, confirmed that two of the strains (from the cerebrospinal fluid of the baby and the vagina of the mother) which were sent to him were group B streptococci and that they belonged to the same antigenic type, type III.

Using commercially available sensitivity testing rings (Mast Laboratories, Ltd, Liverpool), in a disc diffusion test on blood agar, the streptococci were shown to be resistant to streptomycin $(25 \mu \mathrm{g})$, tetracycline $(50 \mu \mathrm{g})$, and to produce sensitivity zones of 20 to $25 \mathrm{~mm}$ in diameter to penicillin $(1.5 \mu \mathrm{g})$, cloxacillin $(5 \mu \mathrm{g})$, ampicillin $(25 \mu \mathrm{g})$, and cephaloridine $(25 \mu \mathrm{g})$. 


\section{TREATMENT}

Intramuscular antibiotic therapy was started at the time of lumbar puncture with initial loading doses of 250,000 units of penicillin and $60 \mathrm{mg}$ of streptomycin; penicillin 100,000 units was repeated six hourly, streptomycin $80 \mathrm{mg}$ eight hourly, and ampicillin $625 \mathrm{mg}$ six hourly. The last convulsion was eight hours later and phenobarbitone was discontinued after two more days; jaundice began to regress and there was general improvement though the temperature did not return to normal for another three days. Culture of cerebrospinal fluid was repeated after five days of antibiotic treatment and was sterile; streptomycin was stopped at this time, penicillin was stopped the next day, and ampicillin two days later; the baby was discharged 16 days after admission, without any abnormal findings and having made a complete recovery. At follow-up clinics one month and three months later, the baby was thriving satisfactorily and showing good weight gain; there was no evidence of any central nervous system abnormality or any other abnormality.

The mother was transferred to an isolation ward and given ampicillin $250 \mathrm{mg}$ six hourly for five days: a high vaginal swab taken at this time did not yield haemolytic streptococci.

\section{ADDITIONAL FINDINGS}

Note was kept during the period July 1968 to July 1969 of instances in which group B streptococci were isolated in this laboratory, either in pure culture or as the predominant organism in a mixed culture. Isolations were obtained from high vaginal swabs in one case of puerperal pyrexia, one case of suspected salpingitis, and in another fivecases of patients presenting with vaginal discharge; in five instances from cord swabs taken as a routine investigation three days after delivery in healthy babies; from skin lesions in two cases with ulcers of the leg and from three cases of skin infections such as infected eczema; from one case of neonatal conjunctivitis.

\section{Isolation of Group B Streptococci from Man in the British Isles}

Dr M. T. Parker has provided information about strains of group B streptococci from human sources in Britain that were submitted to the Streptococcus Reference Laboratory for identification or typing during 1964 to 1967 (personal communication). There were isolates from 29 neonates (Table I); 19 had serious infections and in eight of these cases the same organism was isolated from the mother; 12 of the affected infants died. Clinical information was not received about the remaining 10 newborn babies from whom group B streptococci were isolated.

\begin{tabular}{lr}
\hline Disease & No \\
\hline Meningitis & 8 \\
Septicaemia & 4 \\
Pneumonia & 2 \\
Pericarditis & 1 \\
'Respiratory distress' & 2 \\
Stillbirths & 2 \\
Clinical condition not recorded & 10 \\
Total & 29 \\
\hline
\end{tabular}

Table I Group B streptococci isolated from newborn infants

Isolates were made from 56 adults (Table II); five isolates were from cases of puerperal pyrexia; in two of these the organism was grown from blood cultures and in one instance both mother and baby died of group B streptococcal infection. There were three other isolates from septic infections; from a case of pericarditis, from a case of osteomyelitis, and from an abscess. In the remaining 48 adult cases clinical details are not known; 22 isolates were from the vagina and 26 from other sites.

\begin{tabular}{ll}
\hline Disease & No. of Cases \\
\hline Puerperal pyrexia & 5 \\
Pericarditis & 1 \\
Osteomyelitis & 1 \\
'Abscess' & 1 \\
Not known & 48 \\
Total & 56 \\
\hline
\end{tabular}

Table II Group B streptococci isolated from adults and older children

\section{Discussion}

Group B streptococcal infections of man have been recognized for the past 45 years but until about 1950 it was widely believed that these occurred mainly in the puerperium; there were few reports of neonatal infections. However, studies have been published in the past decade associating group B streptococci to an increasing extent with infections of the newborn. Hood, Janney, and Dameron (1961) recorded isolation of group B streptococci from eight babies born of 66 mothers who had marked perinatal difficulties. Mannik, Baringer, and Stokes (1962) reported a case of group B streptococcal meningitis in an infant with a history of difficult breech delivery. Eickhoff, Klein, Daly, Ingall, and Finland (1964) recorded that of 5,421 live births, 10 newborn babies yielded isolates of group B streptococci, and in seven cases the organism appeared to cause neonatal sepsis; in this series group B streptococci caused more neonatal sepsis than any other single organism. 
Maher and Irwin (1966) reported a single case of meningitis in a 42-hour-old infant, and Winterbauer, Fortune, and Eickhoff (1966) reported two cases of neonatal meningitis due to group B streptococci. There were 20 cases of meningitis of the newborn and four other cases of sepsis due to group B streptococci over a six-year period in the series of Butter and de Moor (1967). Jones and Howells (1968) reported two cases of meningitis, and Rogers (1970) recorded 14 cases of infections due to group B streptococci in newborn infants; the last two reports, unlike those previously mentioned, are the only British publications.

Infections of pregnant and parturient women have been recorded also: Hood and others (1961) reported the isolation of group B streptococci from the genital tract of 16 of 66 women described above as having perinatal problems; Eickhoff and others (1964) recorded 10 isolates of group B streptococci from the genital tracts of cases of septic abortion; in five other cases of women with premature rupture of membranes there was chorioamnionitis, considered to be due to group B streptococci. Butter and de Moor (1967), however, reported only one case of puerperal sepsis due to this organism over a sixyear period and noted that 20 women from whose birth canal group B streptococci had been isolated did not have puerperal infection.

There have been other studies of group B streptococcal infections also, consisting mainly of small numbers of varied cases. For instance, Mannik and others (1962) reported two cases, of pneumonia and subacute bacterial endocarditis, in elderly women. Eickhoff and others (1964) recorded 13 infections in adults over the age of 45 ; eight were diabetics with gangrene of the extremities; three had acute pyelonephritis, and there was a case of unexplained pyrexia and one of suppurative arthritis. Lazarus, Sellers, and Marine (1965) reported a case of meningitis in an elderly woman with chronic pyelonephritis. Butter and de Moor (1967) reported 20 cases of bacteraemia and three of meningitis, mainly in individuals over 60; in 13 fatal cases there was predisposing diabetes or neoplasm.

In the case of group B streptococcal meningitis reported here it seems likely that the site of initial infection was the upper respiratory tract since the organism was recovered from the throat and from venous blood. The same organism was cultured from the mother's vagina 48 hours after delivery: the absence of pyrexia, vaginal discharge, or abnormal lochia suggests that the organism did not cause disease of the mother. The patient was presumably a healthy carrier of group B streptococci; such microorganisms were reported to be part of the normal flora of the genital tract in about $5 \%$ of pregnant women in one study (Hood and others, 1961) and in $9 \%$ of mothers in another (Butter and de Moor, 1967). In the series of Eickhoff and others (1964) the mothers of eight of 10 newborn infants with group B streptococcal bacteraemia had premature rupture of membranes; Butter and de Moor (1967) point out that in group B streptococcal infections of both infants and adults resistance-lowering conditions are frequently present, such as prematurity, hydrocephalus, birth injuries, congenital heart disease, or diabetes. In this case, however, there does not seem to have been a predisposing factor. The infection appears to have been acquired during passage through the birth canal; in the absence of chorioamnionitis it is unlikely that the organism infected the baby in utero by passing through the intact membranes before delivery, as has been suggested by Eickhoff and others (1964).

The baby made a complete recovery but there is a high mortality generally associated with neonatal sepsis due to this organism, as can be seen from the literature (Table III), notwithstanding vigorous antibiotic therapy; antibiotic treatment was started within 36 hours of observation of the first clinical signs at a time when pyrexia was developing; this early initiation of therapy using high dosages of penicillin and ampicillin may have been important factors in recovery.

\begin{tabular}{rcl}
\hline Number of Infants & Deaths & Author \\
\hline 8 & 5 & Hood and others (1961) \\
10 & 4 & Eickhoff and others (1964) \\
1 & 1 & Winterbauer and others (1966) \\
26 & 19 & Butter and de Moor (1967) \\
2 & 1 & Jones and Howells (1968) \\
14 & $14^{1}$ & Rogers (1970) \\
\hline
\end{tabular}

Table III Deaths in newborn infants with Group $B$ streptococcal infections

${ }^{1}$ Five of these were stillborn.

Maher and Irwin (1966) suggested that the eradication of group B streptococci from the birth canal of pregnant women might be valuable in reducing neonatal sepsis. Butter and de Moor (1967) disagreed, however, because they found that although a high proportion of newborn babies yielded isolates of group B streptococci (22 of a series of 252) subsequent illness in these babies due to this organism was rare. A compromise would be reasonable; if group B streptococci were found to be present in the genital tract near to term antibiotic treatment of the baby immediately after delivery would be justified if labour were abnormal or the baby's health in doubt. However, until more is known about the incidence of group B streptococcal infections in the newborn and about the vaginal carrier rate in late pregnancy, 
culture for this organism near to term, as a routine, cannot be advocated.

There is no evidence that transfer of this organism from mother to mother has occurred in maternity units, therefore isolation of such cases specifically as a precaution against outbreaks of infection is probably unnecessary.

There have been few published reports in the British literature since that of Fry in 1938, associating group B streptococci with diseases of man; it is possible that some isolates of group B streptococci are wrongly identified; colonies of this organism on blood agar can resemble Streptococcus viridans and unless biochemical or serological tests are carried out may be reported as such. Growth of both group B and D streptococci occurs on MacConkey's medium; growth on $40 \%$ bile agar is variable and depends on the particular batch of ox bile used. The use of aesculin bile medium on which group B streptococci do not produce hydrolysis of aesculin will help to avoid confusion with group D streptococci.

The findings presented here indicate that this organism causes disease of man in the British Isles more commonly than study of the literature would suggest. Infections of the newborn, associated with a high mortality, are the most serious and occur most frequently; however, infections are not restricted to this age group and involvement of the female genital tract during pregnancy or the puerperium is particularly important because of the danger it represents to mother and child, even when the mother is apparently well; infections of a serious nature, in adults other than such women, occur mainly in the elderly or debilitated and are less common.

I am grateful to Dr J. E. M. Whitehead, Dr M. T. Parker, and Dr C. H. L. Howells for their help and advice, and to the technical staff, Public Health Laboratory, Coventry, for their assistance. I wish to thank Mr H. R. Duval and Dr E. E. Jones, Coventry Maternity Hospital, for permission to publish clinical details.

\section{References}

Butter, M. N. W., and de Moor, C. E. (1967). Streptococcus agalactiae as a cause of meningitis in the newborn, and of bacteraemia in adults. Antonie v. Leeuwenhoek, 33, 439-450.

Eickhoff, T. C., Klein, J. O., Daly, A. K., Ingall, D., and Finland, M. (1964). Neonatal sepsis and other infections due to Group B beta-hemolytic streptococci. New Engl.J. Med., 271, 1221-1228.

Fry, R. M. (1938). Fatal infections by hemolytic streptococcus Group B. Lancet, 1, 199-201.

Hood, M., Janney, A., and Dameron, G. (1961). Beta-hemolytic streptococcus Group B associated with problems of the perinatal period. Amer. J. Obstet. Gynec., 82, 809-818.

Jones, H. E., and Howells, C. H. L. (1968). Neonatal meningitis due to Streptococcus agalactiae. Postgrad. Med. J., 44, 549-551.

Lazarus, J. M., Sellers, D. P., and Marine, W. M. (1965). Meningitis due to the Group B beta-hemolytic streptococcus. New Engl. J. Med., 272, 146-147.

Maher, E., and Irwin, R. C. (1966). Group B streptococcal infection in infancy: a case report and review. Pediatrics, 38, 659-660.

Mannik, M., Baringer, J. R., and Stokes, J. III (1962). Infections due to Group B beta-hemolytic streptococci: report of three cases and review of literature. New Engl. J. Med., 266, 910-913.

Rogers, K. B. (1970). Neonatal meningitis and pneumonia due to Lancefield Group B streptococci. Arch. Dis. Child., 45, 147.

Winterbauer, R. H., Fortune, R., and Eickoff, T. C. (1966). Unusual occurrence of neonatal meningitis due to Group B betahemolytic streptococci. Pediatrics, 38, 661-662. 